\title{
Degradation Model for Erbium-Doped Fiber Amplifiers to Reduce Network Downtime
}

\author{
Christian Merkle \\ Lehrstuhl für Kommunikationsnetze, Fakultät für Elektrotechnik und \\ Informationstechnik, \\ Technische Universität München, \\ Arcisstr. 21, 80333 München, Germany
}

\begin{abstract}
The outage of optical components like optical amplifiers and cross connects reduces the availability of a network and increases the operational expenditures of network operators. Responsible for this is the repair process of optical components which takes very long, because of the large distances in the field. The detection of performance degradation of erbium-doped amplifiers (EDFAs) can be used to reduce the repair time by changing an EDFA before it fails. With the knowledge of the degradation state the remaining life time of an EDFA can be calculated, enabling the operator to plan the replacement of EDFAs to avoid longer outage times. In this paper, an 1,000 km link and a German backbone network are used to simulate the aging of EDFAs with aging curves. Additionally the operational expenditures are considered to compare the cost of the repair process of EDFAs.
\end{abstract}

Keywords: EDFA, aging curve, OpEx.

\section{Introduction}

In today's optical networks EDFAs are widely used because they can amplify many signals on different wavelength at the same time. The most commonly used amplifier in transmission systems is the erbium-doped fiber amplifier (EDFA) 1]. Such amplifiers may fail during their operation, for example due to aging effects of the pump laser, and lead to transmission failure on a link. Due to the high bandwidth which are used on backbone network links today an outage of an amplifier leads to a high loss of data packets transmitted over the failed link. This also has an impact on the revenues of the provider, who has to pay a penalty to the customers affected by the link outage. Hence, network operators monitor their networks to get information of the current network status. If a failure occurs in a backbone network, an alarm is sent from the network management system to the network operator center (NOC). Based on the failure alarms, the operator tries to localize the failure first and solves it afterwards. Today optical amplifiers are not monitored by the management system. The outage of an EDFA takes very long because the technician has to travel to the amplifier to repair it.

In this paper the monitoring of the aging of an EDFA is addressed which enables the operator to plan the repair process and to replace the EDFA before 
it fails. Thus, the repair process can be reduced which increases the network availability. In section 3 the aging of the EDFA performance is discussed and in section 4 two different models, a 1,000 km link and a Germany reference network, are discussed to compare different change strategies for EDFAs. In section 5 the operational expenditures (OpEx) are analyzed for the repair process.

\section{Related Work}

A detailed description of the functionality of an EDFA can be found in [1]. An EDFA amplifies the signal by using a pump signal from a laser, typically at a wavelength of $980 \mathrm{~nm}$ or $1480 \mathrm{~nm}$. But there are several imperfections that a system designer need to worry about when using an amplifier in a system. One disadvantage is the degradation of the performance due to aging of the components. An algorithm how the aging effects of an EDFA can be detected is described in [3]. The basic concept of the algorithm is to calculate the pump diode current that would be needed to create the measured gain at begin of life (BOL) conditions and to compare the results with the actual pump current. A pump laser of an optical amplifier is used to get a constant amplifier gain. With the monitoring of the actual pump current and the data from the data sheet of an amplifier, a network provider has a rough indication of the remaining life time of an amplifier.

In the literature much research is done in the field of fault identification and localization in optical networks as it is presented in [4, 5], and [6]. The first two papers describe different algorithms to detect the location of soft and hard failures. Because the outage time of a network depends also on the time to find a failure, this is a very challenging task, which can reduce the downtime of network links. In [6] the optimal placement of monitoring equipment is described to reduce the number of generated alarms in a network. The goal of all these methods is to identify the components whose failure has caused the received alarms. In our paper we describe also a monitoring concept, but in our concept the degradation of an EDFA is sent to a management system to replace it before it fails. Hence, the repair process of an EDFA can be planned.

The planning of the repair process is important for a network operator to reduce the OpEx of the network. In 7] a methodology to calculate CapEx and OpEx of a telekom operator is described. Different input parameters like staff cost, energy cost, and the description of the repair process and the provisioning process are identified to calculate the OpEx of a German reference network. The OpEx calculation for the German reference network indicates that the repair processes are the second largest cost factor of the OpEx of a network. Hence, with the optimization of the repair process, the OpEx of a telecom operator can be reduced.

In [8] a total cost of the network ownership model is presented. The paper describes the different factors which contribute to the capital expenditures and operational expenditures. The OpEx are defined as the cost related with the operation of a network. Several types of cost are described which can be 
distinguished for the reparation sub-process of a network failure. These factors are used in section 5 when the cost of the EDFA repair process are described.

\section{EDFA Degradation Model}

In this section the degradation of the pump current of an EDFA and the dimensioning of the pump current threshold are described. As mentioned previously, every amplifier requires a pump laser to bring the erbium ions into a higher energy band to amplify an incoming WDM signal [1]. Due to aging effects of the amplifier, the pump power of the amplifier has to be increased to obtain a constant gain. In this paper the pump current is used for the description of the pump power. The aging of an EDFA can be described by aging curves as shown in Fig. 1] The curves show the necessary pump current of an EDFA over a certain time period to achieve the same amplifier gain. There are different aging curves because not every EDFA has the same aging characteristic. It is possible that some EDFAs degrade faster than others. The second characteristic of the aging curves is that they have no constant gradient over the life time of an EDFA. At the begin of life the pump current gradient is higher than at the end of life. The begin of life pump current shown in Fig. 1 was taken from a data sheet of an EDFA. The used pump current of the EDFA at the begin of life is $550 \mathrm{~mA}$. Beside the BOL pump current an EDFA has also a maximum pump current up to which it can be operated without it fails. This parameter is also given by the data sheet of a manufacturer of an EDFA. Here, the maximum pump current which can be used is $670 \mathrm{~mA}$. With the algorithm described in 3] the actual needed pump current can be calculated. With this knowledge the pump current threshold which indicates that the amplifier has to be replaced can be calculated as the difference between the BOL current and the actual pump current. The

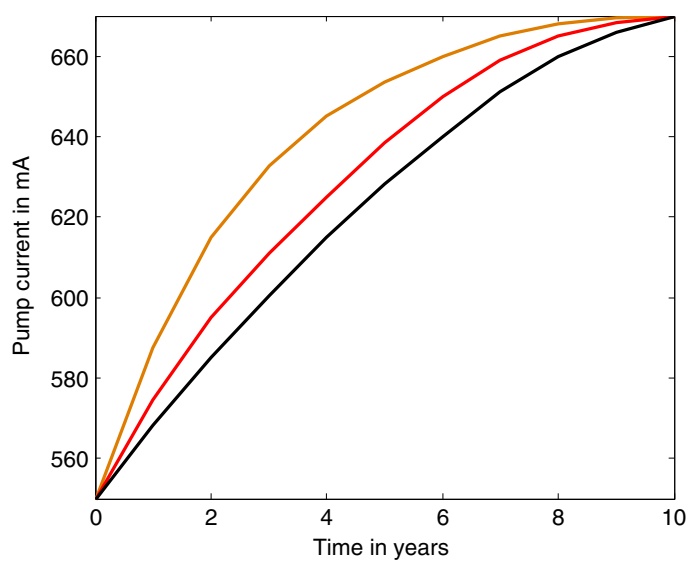

Fig. 1. Different aging curves of EDFAs 
network operator has to define the pump current threshold for the EDFAs. The value of the threshold depends on the remaining life time an operator wants to have for the EDFAs. When an EDFA reaches the threshold an alarm message is send to the network operation center (NOC) to inform the operator that it has to be replaced.

The advantage of having a remaining life time for an EDFA is that the network operator does not need to change the EDFA immediately and can plan the repairing process. If an EDFA has a remaining life time of one year, for example, the operator can wait if further EDFAs reach also their degradation threshold in a certain time period after the first EDFA signals its degradation state. Hence, the repair team in the field has the possibility to repair more than one EDFA in one shift. Also the network availability is increased because the traveling time of the repair team does not account to the outage time of an EDFA anymore. Considering only the link breaks is not sufficient to analyze the availability of a backbone network. Also the repair time of the EDFAs and, hence, the downtime of the link are important factors for the network availability. An analysis of the repair time and link breaks is presented in section 4.

Another goal for the operator is to reduce the OpEx of backbone networks. One part of the OpEx is the repair process of failed network components. If the repair time of an EDFA can be planned by an operator, the traveling time can be optimized which reduces the traveling overhead and also the cost of the repair process. Further cost savings can be achieved due to the reduction of spare parts and stock size. The knowledge about the remaining life time of an EDFA can be used to order a new unit not until an old unit reaches the degradation threshold. Hence, only a minimum of spare parts is needed to repair an unforeseen outage of an EDFA for example. The OpEx of the repair process are discussed in section 5.

The signaling of the degradation threshold of an EDFA can be done with a network management protocol like the Simple Network Management Protocol (SNMP). All EDFAs in a network periodically send their actual pump current to a NOC. If an EDFA reaches its degradation threshold, it sends an additional message to the NOC to inform the operator that the degradation threshold was reached. The time interval of the EDFA aging messages are not constant over the EDFA life time. At the begin of life the management messages are sent in a larger interval and when the actual pump current is closer to the degradation threshold the management messages are sent more frequently.

\section{Simulation Setup and Results}

In this section two different simulation scenarios are analyzed. First, a link with a length of $1000 \mathrm{~km}$ is considered to simulate two different strategies to replace degraded EDFAs. In the second simulation scenario, a German 50 node reference network is used to analyze the repair process in a larger network. For both scenarios an EDFA spacing of $80 \mathrm{~km}$ is used. To simulate the aging of the pump current of an EDFA the curves in Fig. 1 are used. In the beginning of the simulation, one of the aging curves is randomly assigned to every EDFA. The 
replacement of an EDFA corresponds to a random allocation of a new aging curve. The simulations and the analysis of the results are done with Matlab. For every EDFA model 10 independent iterations have been conducted to avoid statistical aberrations. The main simulation parameters are shown in table 1 .

Table 1. Simulation parameters

\begin{tabular}{l|c|c}
\hline Simulation parameters & 1000km link & German 50 node network \\
\hline FIT rate of EDFAs & 2850 & 2850 \\
Degradation curves of EDFAs & 5 & 5 \\
Used EDFAs & 12 & 241 \\
Simulated time period in years & 30 & 100 \\
Simulation runs & 10 & 10 \\
\hline
\end{tabular}

\subsection{Consideration of a 1000km Network Link}

For the analysis of the $1,000 \mathrm{~km}$ link 12 EDFAs are used. These 12 EDFAs do not have the same aging process and, hence, degrade in different rates. The fiber at the link also has a failure rate which is given in failure in time (FIT). For the simulations $380,22 \frac{\mathrm{FIT}}{\mathrm{km}}$ are used for the fiber. One FIT means one failure per $10^{9}$ hours. It is assumed that two repair teams are at one NOC which is located in the middle of the link at $520 \mathrm{~km}$. Hence, every repair team has to maintain one half of the $1,000 \mathrm{~km}$. The spare parts are also located at the NOC, hence, there is no additional traveling to a stock required. In the following two different replace strategies are analyzed for the $1,000 \mathrm{~km}$ link.

In the first simulation scenario, only the EDFAs which reached the degradation threshold are changed either at the end of every year or if a fiber fails. If the link is down due to a fiber failure, it is expedient to also replace all EDFAs which received the degradation threshold because further downtimes due to EDFA changes are avoided. For the simulations a time period of 30 year is considered to determine the average time period between two replaced EDFAs over a certain time period. In Fig. 2 the total number of changed EDFAs and the number of link breaks in 30 years are shown. Three different pump current thresholds were taken for the simulations. In average 67 EDFAs have to be changed in 30 years when a threshold of $620 \mathrm{~mA}$ for the pump current is used. The replacement of these 67 EDFAs lead to 25 link breaks in 30 years which means that there was a link break almost yearly. If a threshold of $660 \mathrm{~mA}$ is used for the pump current 4 link breaks occur in 30 years and lead to 13 replaced EDFAs. As expected the higher threshold leads to less replaced EDFAs and, hence, to less link breaks. The disadvantage of a higher threshold is that the rest life time of the EDFAs is shorter compared to threshold of $620 \mathrm{~mA}$. Because the operator does not know how the aging curve of the EDFA exactly looks like, he can only determine a time interval when the EDFA will fail with a certain probability.

A further change strategy of the EDFAs was simulated to compare it with the results of the first scenario. In the second scenario all EDFAs at the link 

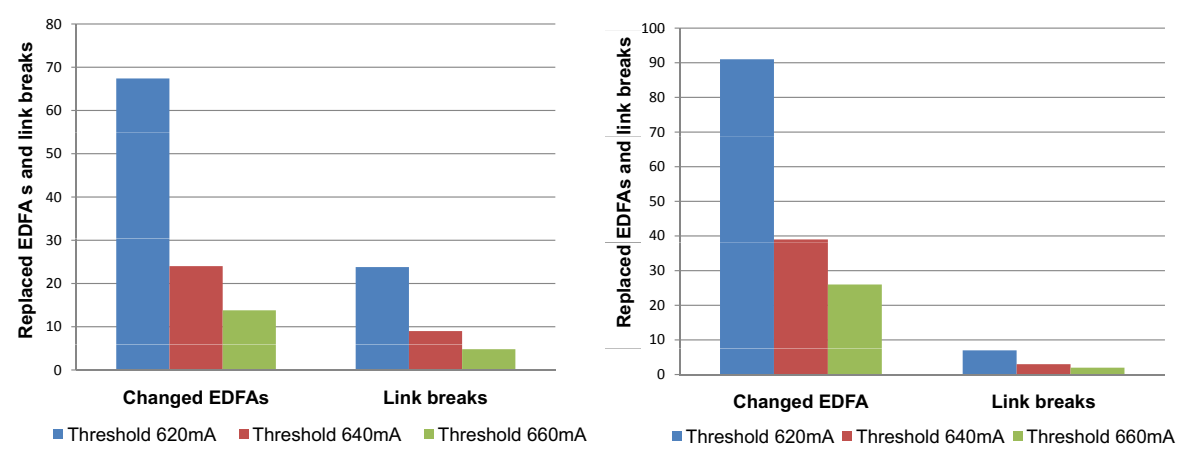

Fig. 2. Number of EDFA outages and link Fig. 3. Number of EDFA outages and link breaks for scenario 1 breaks for scenario 2

are changed if one EDFA reaches the degradation threshold or the fiber fails. In Fig. 3 again the average number of replaced EDFAs and the number of link breaks are shown. For a threshold of $620 \mathrm{~mA}$, the average number of changed EDFAs is 84. Compared with the values of the first scenario it can be seen that, as expected, more EDFAs were changed in 30 years. The advantage of the second change strategy appears if the number of link breaks is considered. The 84 replaced EDFAs lead to 7 link breaks. Hence, in the second scenario the number of link breaks is reduced about $72 \%$ in comparison to the first scenario. The number of link breaks are further reduced if the threshold is increased. For a threshold of $660 \mathrm{~mA}$ the number of link breaks is 2 .

These two cases illustrate that the replacement of more than one EDFA on one link at the same time can reduce the number of link breaks. Hence, if one EDFA has reached its degradation threshold it can be an advantage also to replace other EDFAs on the same link. Therefore, the current value of the threshold of the other EDFAs on the same link can be an indication which of the EDFAs the operator should replace additionally.

To further evaluate the different replace strategies the repair time to replace degraded EDFAs is calculated. For both scenarios, a degradation threshold of $620 \mathrm{~mA}$ is assumed. In the first scenario only the EDFAs are replaced which have reached their degradation threshold. The repair time $t_{\text {Repair }}$ for both scenario is given by the maximum of the repair times of both teams and the travel time $t_{E D F A_{X}-E D F A_{Y}}$ between the EDFAs.

$$
t_{\text {Repair }}=\max \left(t_{\text {RepairTeam } 1}, t_{\text {RepairTeam } 2}\right)+t_{E D F A_{X}-E D F A_{Y}}
$$

The repair time of each EDFA is $2 \mathrm{~h}$, as described previously. The traveling time to the first EDFAs can be neglected for both scenarios because it is assumed that the link is disabled not until the repair team has arrived the first EDFA. The travel time $t_{E D F A_{X}-E D F A_{Y}}$ depends on the distances between the EDFAs that have to be replaced. For the first scenario, an average distance of $200 \mathrm{~km}$ was obtained. Hence, the repair time $t_{\text {Repair }}$ is $171.42 \mathrm{~h}$ in 30 years. When all EDFAs 
are changed if one or more EDFA have reached their degradation threshold, the total repair time is $126 \mathrm{~h}$. In this model the travel distance is constant and has a length of $420 \mathrm{~km}$. The result shows that the repair time and also the downtime of the link is reduced by $26.5 \%$ in comparison to the first scenario. Hence, the availabilty of the network can be increased if always all EDFAs are changed on one link. Besides the downtime also the OpEx of network has to be considered. The OpEx of the two scenarios is discussed in the next section.

\subsection{Germany 50 Node Reference Network}

In this section the Germany 50 node reference network 11] is used to compare the EDFA degradation model with a FIT rate model. The reference network consists of 50 nodes and 87 links and has an average link length of $131 \mathrm{~km}$. The total link length of the network is $8,874.13 \mathrm{~km}$ and the number of used EDFAs is 241 . There are two NOCs in the network, each responsible for half of the 241 EDFAs. For the simulations a time period of 100 years was used to analyze the total number of replaced EDFAs and the number of EDFAs which can be repaired at the same shift. The difference between the two models is the time when an EDFA is replaced. By the FIT rate model the EDFAs are replaced after they failed as it is done in networks today. We take into account a time period of 100 years to avoid transient effects of the simulations of the degradation model. In this model all EDFAs are considered as new installed equipment in the network which is not true in a real network. Hence, the analysis of the results is done after 10 years, when the first EDFAs are changed. The following assumptions are used for both models: the number of required technician to repair an EDFA is 1 and it takes 2 hours to repair and test an EDFA [7].

Simulation with FIT rate. In this model the number of EDFA outages for a EDFA FIT rate of 2,850 2] are simulated. The simulation results of this model serve as reference values for the EDFA degradation model which is analyzed in the next section. The failure probability of an EDFA in the time interval $t$ is given by:

$$
P\left(T_{\text {out }} \leq t\right)=1-\exp \left(-\lambda_{F I T} \times t\right)
$$

Hence, the probability that exact one EDFA fails within one year is $2.47 \%$ and the probability that at least one EDFA fails within one year is $99.76 \%$. This failure probabilty leads to 5.77 failed EDFAs per year in average. The average repair time is calculated with equation 3

$$
\bar{t}_{\text {Repair }}=\frac{\sum_{k=1}^{N}\left(t_{N O C-E D F A_{k}}+t_{\text {repair }}\right)}{N}
$$

and is $6.69 \mathrm{~h}$ for each EDFA. $\mathrm{N}$ is the number of failed EDFAs, $t_{N O C-E D F A_{k}}$ is the traveling time from the NOC to the $E D F A_{k}$, and $t_{\text {repair }}$ is the repair and test time of an EDFA. With this values a whole repair time of $3,857.97 \mathrm{~h}$ in 100 years is obtained. 
Simulation with degradation threshold. By using the pump current threshold described in section 3. an EDFA can be changed before it fails. This allows the planning of the repair process and the reparation of more than one EDFA in one working shift. This means that a repair team can plan a route between the NOC and the EDFAs they have to repair. The goal of planning the repair process is to repair as much EDFAs as possible without traveling back to the NOC. An example for a repair process with two degraded EDFAs is illustrated in Fig. 4 and in Fig. (5 In Fig. 4 the two EDFAs are repaired independently from

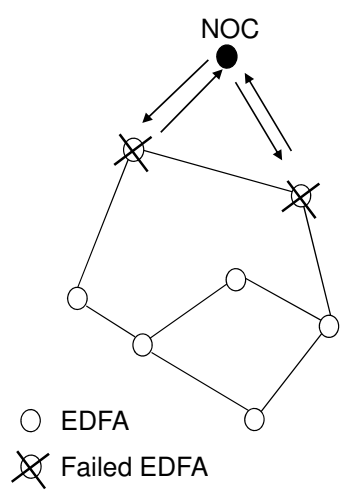

Fig. 4. EDFAs repaired independently

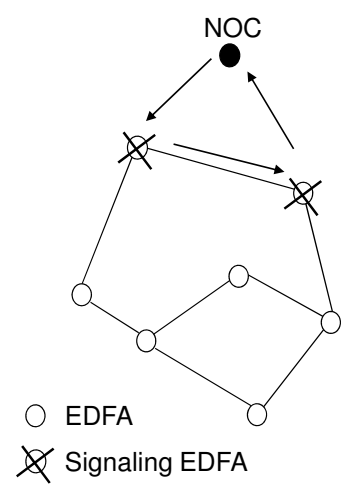

Fig. 5. Two EDFAs repaired in one shift

each other. The repair team must always travel from the NOC to the EDFA and back. This is the case in the FIT rate model described above. In Fig. 5 the repair process was planned in advance and the two EDFAs can be repaired by one repair team in one cycle. In order that it is worth to repair the two EDFAs in on cycle the travel time $t_{\text {travel }}$ must be shorter than the sum of the single traveling times to the single EDFAs as shown in Fig. 4. The repair time for an EDFA is the same for both cases and can be neglected. The traveling time for

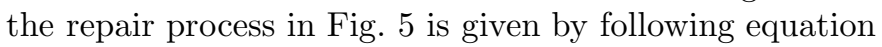

$$
t_{\text {travel }}=t_{N O C-E D F A_{x}}+x \times t_{E D F A_{x}-E D F A_{y}}+t_{E D F A_{y}-N O C}
$$

The whole travel time $t_{\text {travel }}$ of the technician is the sum of the traveling time from the NOC to the first EDFA $t_{N O C-E D F A_{x}}$, the traveling time between the EDFAs $t_{E D F A_{x}-E D F A_{y}}$, and the traveling time from the last EDFA back to the

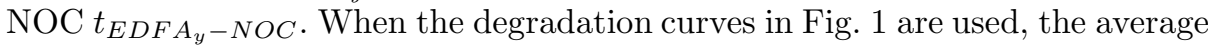
total number of replaced EDFAs is 7.2 per year and in $42 \%$ of the 100 years it was possible to repair two EDFAs in one shift. In the other years, the distance between the replaced EDFAs is to large to repair it in the same shift. The results are shown in table 2 .

For the degradation model, the number of replaced EDFAs is higher then for the FIT rate model. This is caused because the EDFAs are replaced before they 
Table 2. Major results for the German reference network

\begin{tabular}{l|c|c|r}
\hline Parameters & FIT rate model & Degradation model & \\
\hline Outage probability & 2850 & aging curves & - \\
Replaced EDFAs per year & 5.77 & 7.2 & - \\
Total travel time & $2703.97 \mathrm{~h} / 100$ years & $3036.43 \mathrm{~h} / 100 y e a r s$ & $+10.95 \%$ \\
Total repair time & $3857.97 \mathrm{~h} / 100$ years & $1546 \mathrm{~h} / 100$ years & $-60.03 \%$ \\
\hline
\end{tabular}

fail. A further result is that it was only possible to repair two EDFAs in one shift because of the large distances between a NOC and the EDFA. If more than two NOCs will be used for the simulation it should be also possible to repair three EDFAs in one shift. The small number of NOCs is also a reason for the small number of years in which two EDFAs can be repaired in one shift. The advantage of the degradation model can be seen by considering the repair times of the EDFAs. Because the EDFAs are changed before they fail the traveling time does not account to the total repair time. Hence, the total repair time for the EDFAs is only the repair time of the replaced EDFAs and is $1544 \mathrm{~h}$ per 100years. This means that the repair time and hence, the downtime of the network is reduced by $60 \%$ in comparison to the FIT rate model. But in the degradation model, the traveling time is higher because more EDFAs had to be replaced than for the FIT rate model. As described above it was possible to repair two EDFAs in one shift in $42 \%$ of the 100 years and this lead to a reduced travel distance of $26,520 \mathrm{~km}$. With an average speed of $70 \frac{\mathrm{km}}{\mathrm{h}}$ the saved traveling time is $378.86 \mathrm{~h}$. Hence, the whole travel time is $3,036.43 \mathrm{~h}$ per 100 years for the degradation model. Thus, the travel time is increased by $10.95 \%$.

\section{OpEx Analysis of the Repair Process}

In this section the operational expenditures $(\mathrm{OpEx})$ of the repair process of the EDFAs are discussed. For the OpEx calculations, only the Germany reference network is considered. Important for the cost calculation are the basic salary of a technician, the repair time for an EDFA, and the traveling time in the field. For the calculation, it is assumed that the stock is located at the NOC and the repair team must not drive there to collect the spare parts. In the following, the FIT rate model and the degradation models are analyzed.

\subsection{OpEx Analysis for EDFA Outages Given by the FIT Rate}

To calculate the cost of the repair process following equations are used:

$$
\begin{gathered}
c_{\text {Repair }}=\left(c_{\text {Salary }} \times t_{\text {repair }}\right)+c_{E D F A}+c_{\text {stock }} \\
C_{\text {Stock }}=0.05 \times P_{E D F A} \times N_{E D F A}
\end{gathered}
$$

The cost of the repair process is the sum of the cost for the technician, the cost of the EDFA, and the cost of the stock. Further cost like gas are neglected for OpEx 
calculations. The basic salary of a technician is assumed to be US $\$ 36.19$ per hour. This value is based on a network technician salary per year of US $\$ 37,000$ [10]. The cost of an EDFA is US $\$ 11,850[7$ and the cost of maintaining the stock is assumed to be $5 \%$ of the equipment cost and is given in eqn. 6. How much the operator has to pay to maintain the stock depends on the number of EDFAs in the stock and the price of an EDFA. The analysis of the FIT model has shown that the repair time is $3,857.97 \mathrm{~h}$ and that 557 EDFAs had to be replaced per 100 years. Because in average 6 EDFAs are replaced per year it is assumed that 6 backup EDFAs are in the stock. With these values and the equations above the total cost of the repair process are US $\$ 70,955.69$ per year.

\subsection{OpEx Analysis for the EDFA Degradation Concept}

For the degradation model the same equations are used to calculate the cost of the repair process. It is assumed that only one EDFA is in the stock because the operator has enough time to order a new EDFA if one needs to be replaced. With the values obtained by the analysis of repair time, the cost of the repair process are US $\$ 87,570.15$ per year. As it can be seen from the results, the OpEx of the FIT rate model are minor then those of the degradation model. But it has to be mentioned that the penalties that a operator has to pay if he cannot fulfill the service level agreements are not included in the OpEx calculations. Furthermore, the selected pump current threshold for the simulations was $620 \mathrm{~mA}$ which means that the EDFAs have in average a rest life time of 6 years. If a higher threshold is used the OpEx for the degradation model should be minor.

\section{Conclusion}

A degradation concept for EDFAs has been presented that allows the change of EDFAs before they fail. Every EDFA has a specific aging curve of its pump current which is used to determine the remaining life time of an EDFA. The remaining life time depends on the pump current threshold which is set by the network operator. The evaluation of the link model shows that the replacement of all EDFAs on one link reduces the downtime of the link by $26.5 \%$. Furthermore, the degradation model was compared with a FIT rate model of EDFAs where the EDFAs are only replaced when they fail. This was done by using a Germany reference network. The results show that for the degradation model the downtime of network links can be reduced by $60 \%$ because the traveling time to the EDFAs can be neclected. But the former change of the EDFAs leads to a higher number of changed EDFAs in comparison to the FIT rate model. Finally, the OpEx for the repair process of the Germany reference network are analyzed. The results show that the repair cost of the degradation model is higher, but service level agreements penalties were not considered in the calculation which increase the OpEx of the FIT model.

Acknowledgement. The author is grateful to Lutz Rapp (Siemens AG) for insightful discussions about the aging of an EDFA. 


\section{References}

1. Ramaswami, R., Sivarajan, K.N.: Optical Networks - A Practical Perspective. Morgan Kaufmann Publishers, San Francisco (2002)

2. Mello, D.A.A., Schupke, D.A., Scheffel, M., Waldman, H.: Availability Maps for Connections in WDM Optical Networks. Design of Reliable Communication Networks (2005)

3. Rapp, L.: Quality Surveillance Algorithm for Erbium-Doped Fiber Amplifiers. Design of Reliable Communication Networks (2005)

4. Mas, C., Thiran, P.: An efficient algorithm for locating soft and hard failures in WDM networks. IEEE Journal on selected Areas in Communications 18(10), 1900-1911 (2000)

5. Mas, C., Nguyen, H.X., Thiran, P.: Failure location in WDM networks. Optical WDM Networks: Past Lessons and Path Ahead (2004)

6. Stanic, S., Subramaniam, S., Choi, H., Sahin, G., Hyeong-Ah, C.: Efficient alarm management in optical networks. In: DARPA Information Survivability Conference and Exposition (2003)

7. Verbrugge, S., Colle, D., Pickavet, M., Demeester, P., Pasqualini, S., Iselt, A., Kirstädter, A., Hülsermann, R., Westphal, F.J., Jäger, M.: Methodology and input availability parameters for calculating OpEx and CapEx costs for realistic network scenarios. Journal of Optical Networking 5, 509-520 (2006)

8. Machuca, C.M.: Expenditures Study for Network Operators. Transparent Optical Networks 1, 18-22 (2006)

9. Zhang, K.J., Li, S.Y., Liou, L.W., Corless, J.D., Dominguez, M.: Bandwidthexpandable erbium-doped fiber amplets. IEEE Photon. Technol. Lett. 13(4), 281-283 (2001)

10. Payscale: Salary Survey for Job: Computer/Network Support Technician, http://www.payscale.com/research/US/Job=Computer_\%2f_Network_Support_ Technician/Salary (last seen 2010)

11. SNDlib: Germany 50 node reference network, http://sndlib.zib.de/home.action (last seen 2010) 